\title{
Zur Erforschung des Quartärs in der Schweiz
}

Das Quartär mit einer Dauer von vielleicht 2 Millionen Jahren ist die jüngste und kürzeste Periode unserer Erdgeschichte und zeichnet sich aus durch die Eiszeiten und die Entwicklung des Menschen. Obwohl das Quartär die jüngste Geschichte der Erde umfaßt, sind zahlreiche grundlegende Fragen der Dauer (Grenze Tertiär/Quartär) und der zeitlichen Gliederung noch weitgehend ungeklärt. Die vorhandenen Profile enthalten oft nur zufällige Zeitmarken. Es ist daher verständlich, daß viele Disziplinen der Natur- und Geisteswissenschaften an der Quartärforschung beteiligt sind. Die vorliegende Übersicht berücksichtigt hauptsächlich die Eiszeitforschung und kann daher nicht eine erschöpfende Darstellung sein.

\section{Historisches zur Erforschung des Eiszeitalters}

K. KAISER hat 1975 die Geschichte der Eiszeitforschung von ihren Anfängen bis in die neuere Zeit zusammenfassend dargelegt. Folgt man diesem deutschen Kollegen, einem guten Kenner der schweizerischen Quartärablagerungen, so liegt die Wiege der Eiszeitforschung in der Schweiz. Er schreibt: «Aus dem Befund, da $\beta$ von den Blöcken im Schweizer Molasseland viele aus dem Wallis stammen, schloß wohl erstmalig B.F.KUHN (1787) in seinem "Versuch über den Mechanismus der Gletscher" auf eine weit ausgedehntere Alpenvergletscherung. Die Walliser Bergführer J. P. PERRAUDIN aus Lourtier und M. DEVILLE aus Chamonix äußerten 1815 unabhängig voneinander ebenfalls die Ansicht einer früher sehr viel größeren Gletscherausdehnung, worauf Gletscherschliffe auf nackten Felsoberflächen und Blocktransporte ins Alpenvorland $\mathrm{zu}$ begründen wären.» J. J. SCHEUCHZER (1672-1733) war einer der ersten, die sich wissenschaftlich mit Gletschern beschäftigten (1708: "Naturgeschichte des Schweitzerlandes»). H.B.DE SAUSSURE (1740-1799) erweckte mit seinem Werk "Voyages dans les Alpes» in weiten Kreisen das Interesse für die Alpengletscher.

Es ist das Verdienst des Walliser Forstingenieurs IGNAZ VENETZ, Sitten (1788-1859), die Vorstellungen einer allgemeinen Vereisung gefördert und die Auffas-

\footnotetext{
'Schweizerische Naturforschende Gesellschaft
}

sung des Polyglazialismus erstmals verfochten zu haben. So berichtete er 1816 über den Gletschertransport von Felsblöcken im westalpinen und norddeutschen Raum. Auf ein Preisausschreiben der SNG ${ }^{1}$ hin berichtete er 1821 über die Schwankungen und den einstmals viel größeren Stand der Alpengletscher. Die Moränen des Schweizer Mittellandes und die Findlinge bis zum Jura-Südfuß erklärte er als Ablagerungen vorzeitlicher Walliser Gletscher $(1829,1830)$.

JEAN DE CHARPENTIER (1786-1855), Direktor der Salzbergwerke von Bex (Waadt), trug 1834 der SNG in Luzern die Theorie einer diluvialen Vergletscherung für den gesamten Alpenraum vor. LouIS AGASSIZ (1807-1873) begründete 1840 - damals Professor in Neuchâtel - die Vorstellung einer pleistozänen Vergletscherung der Nordhalbkugel und verhalf der Vergletscherungstheorie weltweit zum Durchbruch ("Études sur les glaciers»).

Praktisch gleichzeitig reifte die Idee von mehreren Eiszeiten: I. VENETZ schloß wohl als erster 1822 aus zwischen Moränen gelegenen Ligniten bei Thonon am Genfer See auf eine mehrmalige Vergletscherung. Der Schweizer Geologe und Prähistoriker A. DE MORLor (1820-1867) förderte aufgrund der schotterstratigraphischen Befunde bei Thonon die Auffassung von I. VENETZ, wonach mehrere Eiszeiten, getrennt durch warme Perioden, aufeinander gefolgt seien. Er prägte auch den im heutigen Sinne verwendeten Begriff "Quartär».

OSWALD HEER (1809-1883), Botanik-Professor in Zürich, konnte 1858-1865 die polyglazialistische Auffassung durch paläobotanische Studien an zwischen Moränen gelegenen Schieferkohlen bei Uznach, Dürnten und Gossau sowie der fossilen Flora von St. Jakob an der Birs untermauern, wobei der Begriff "Interglazial» eingeführt wurde.

Weitere Belege stammten von s.GRAS (1856-1857, Rhonetal), J.C. DEICKE (1858, Mörschwil bei St. Gallen) und F. MÜHLBERG (1896 im Aargau).

F. MÜHLBERG lieferte mit seinen Karten (1901, 1904, $1908,1910,1913$ ) auch einen bedeutenden Beitrag zur Verbreitung der Gletscher und ihrer Schotterfluren.

Dr. Conradin A. Burga, Prof. Dr. Gerhard Furrer Geogr. Institut der Universität Zürich, Postfach, 8033 Zürich 
Als ALBERT HEIM (1849-1937) 1869 das Diplom als Fachlehrer für Naturwissenschaften an der ETH erhielt, hatte er zuvor von seinem Lehrer ARNOLD ESCHER VON DER LINTH folgendes Thema zUr Aufgabe erhalten:

«1) Welche Wirkungen üben die Gletscher aus? Und in Folge welcher Eigenschaften?

2) Finden sich außerhalb dem Gebiet der jetzigen Gletscher Erscheinungen, welche mit Sicherheit auf eine einst größer gewesene Verbreitung von Gletschern hinweisen?»

Die 180 Manuskriptseiten umfassende Arbeit diente als Grundlage zum 1885 erschienenen "Handbuch der Gletscherkunde», wo das gesamte damalige Wissen über Gletscher zusammengefaßt wurde. Es folgten weitere Gletscher- und Eiszeitstudien, so z. B. 1919 in der "Geologie der Schweiz» und der Aufsatz «Über das absolute Alter der Eiszeit" (1894).

Im grundlegenden Werk "Die Alpen im Eiszeitalter» (1901-1909) führten A.PENCK (1858-1945) und E. BRÜCKNER (1862-1927) die Eiszeit-Begriffe "Günz, Mindel, Riß, Würm» ein und schufen somit die Grundlage für eine weltweite Gliederung des Eiszeitalters.

Zuvor veröffentlichte der Engländer J. GEIKIE 1874 das Standardwerk "The Great Ice Age».

Die Kontroverse Monoglazialismus/Polyglazialismus fand erst 1913 mit der Errichtung des von O. AMPFERER angeregten Lepsius-Stollens unter die Höttinger Brekzie nördlich Innsbruck ein Ende.

1949 erschien das A.HEIM und A.PENCK gewidmete "Handbuch der Gletscherkunde und Glazialgeologie» von R. von KLEBELSBERG. Die neueste und für die Schweiz ausführlichste Darstellung des Eiszeitalters stammt von R. HANTKE $(1978,1980,1983)$.

Im Jahre 1987 wird anläßlich der Jahrestagung der SNG in Luzern - in Zusammenarbeit mit dem Gletschergarten - Bilanz über 200 Jahre Eiszeitforschung in der Schweiz gezogen werden.

\section{Das Landeskomitee fuir die INQUA ${ }^{2}$ der SNG}

Wie in den Anfängen der Erforschung des Eiszeitalters Gelehrte verschiedener Disziplinen ihre Erkenntnisse zur Aufhellung vergangener Gletscher- und Klimazustände beitrugen, stammen auch die jüngsten Beiträge zur Quartärforschung von Vertretern verschiedener Fachrichtungen. Um in der Schweiz eine gute Koordination zwischen diesen zu gewährleisten und um die Verbindungen mit ausländischen Fachgesellschaften zu pflegen, wurde im Jahre 1975 auf Initiative des Geologen Prof. w. K. NABHOLZ, Bern, das Landeskomitee für die INQUA gegründet. Ihm gehören vorwiegend Hochschullehrer, aber auch Mitarbeiter geologischer Bureaus an. Gegenwärtig sind die folgenden Disziplinen vertreten: Geologie, Botanik, Paläontologie, Geographie, Urgeschichte und Physik.

\section{Zu den einzelnen Disziplinen im Dienste der Quartärforschung}

\subsection{Geologie}

Mit den Ereignissen, die zur Beschreibung des ersten lithostratigraphischen Profils im Quartär der Schweiz geführt haben, zeichnen sich grundlegende Beziehungen $a b$, die für die Eiszeit-Forschung in unserem Lande von Bedeutung sein sollten: Es ist dies die enge Wechselbeziehung zwischen angewandter oder technischer Geologie der Lockergesteine und der stratigraphisch-wissenschaftlich orientierten Betrachtungsweise. - Im frühen 18. Jahrhundert hat der Zürcher Arzt und Naturforscher J.J.sCHeuchzer das damals größte Bauvorhaben seiner Zeit besucht, nämlich den Durchstich des Strättlighügels bei Thun zur Korrektur des Kanderlaufes (1711-1714). Dabei ist scheuchzer auf die Schieferkohlen im Glütschtal aufmerksam gemacht worden und hat sie im "Herbarium Diluvianum» (1723) mit dem Liegenden und Hangenden als erstes eiszeitstratigraphisches Profil - freilich noch vor dem Hintergrund der Sintflut-Ideen - beschrieben.

In einer weiteren glazialgeologischen Grundsatzdiskussion von der letzten Jahrhundertwende unter dem Motto "Glaziale Tiefenerosion, ja oder nein?" war es wiederum die angewandte Geologie, die, diesmal auf tragische Art und Weise, eine Klärung der Diskussion bewirkte. Mit dem Schutt-Einbruch im Lötschberg am 24. Juli 1908 ist die Existenz übertiefter Talabschnitte bewiesen worden.

Im gleichen Zeitpunkt sind erste quartärgeologische Monographien erschienen (L.DU PASQUIER 1891, A. AEPPLi 1894, A. BALTZER 1896, J. OBERHOLZER 1900, F. A. FOREL 1904, J.FRÜH und C. SCHRÖTER 1904, O. FREY 1907, R. FREI 1912, E. BÄRTSCHI 1913).

F. HOFMANN $(1951,1957,1963,1973)$ hat die von C. FALKNER (1909), A.LUdWIG $(1916,1930)$ und F.SAXER $(1949,1960,1965,1968)$ begonnenen quartärgeologischen Untersuchungen im Raum St. Gallen fortgeführt und in der Schweiz erstmals den allerødzeitlichen Laachersee-Bimstuff nachgewiesen.

Die weiteren quartärgeologischen Mitteilungen bis in die neuere Zeit hinein sind praktisch ausschließlich aus der wissenschaftlichen Interpretation praktischer Erfahrungen aus der Lockergesteinsgeologie hervorgegangen und haben zugleich eine gewisse Unabhängigkeit vom PENCK-BRÜCKNERSchen System bewahrt (J.HUG 1919, P. BECK, 1933, A.VON MOOS 1943, H. JÄCKLI 1962, A. JAYET 1965, C. SCHINDLER 1968).

Methodisch erweiterte Aspekte sind durch die Arbeiten von J.P.PORTMANN (1955) und U.GASSER u. w. K. NABHOLZ (1969) in die Diskussion gekommen, und aktuogeologisches Gedankengut ist neuerdings mit den Seenprojekten der Universitäten Genf

\footnotetext{
${ }^{2}$ Internationale Quartär-Assoziation, eine in ihren Anfängen bis 1926 zurückgehende Organisation
} 
(J.P. Vernet), Bern (A.MAtTer) und der ETH in Zürich (K. HSÜ, K. KELTS), wenigstens für quartärgeologische Teilbereiche, erarbeitet worden (vgl. z.B. C. SCHINDLER 1976).

Die Großbaustellen und die Kiesentnahmestellen während der sechziger und siebziger Jahre in der Umgebung von Bern haben es C. SCHLÜCHTER (1976) und F. DIEGEL $(1975,1976)$ erlaubt, unter Verwendung stratigraphischer Klassifikationsprinzipien, verbunden mit modernen sedimentologischen Methoden, eine Regionalstratigraphie gewissermaßen als Neubeginn zu erarbeiten. - Seit vielen Jahren laufen großräumige hydrogeologische Untersuchungen in den Haupttälern des Mittellandes, deren Ergebnisse meist überraschend komplex und oft schwer zu deuten sind (L. u. G. WYSSLING 1978, T.LOCHER U. M.FREIMOSER 1980, C. SCHINDLER, H. RÖTHLISBERGER U. M. GYGER 1978). F. HOFMANN (1977) versuchte aufgrund von geologischen Daten und Geröllanalysen eine Deutung der Talgeschichte im Raume Schaffhausen-Thurtal-Rafzerfeld. Die gegenwärtig laufenden hydrogeologischen Untersuchungen werden weitere quartärgeologische Zusammenhänge aufdecken (P. KEllerhals u. B. TRÖHLER 1976).

\subsection{Geomorphologie, Dendrochronologie und Klimatologie}

Der Berner Geograph B. MESSERLi und seine Schüler konzentrieren sich auf die Klimageschichte der Schweiz seit dem Mittelalter; eine Datenbank verschiedenster Klima-Parameter ist im Aufbau begriffen (vgl. auch C. PFISTER, im Druck). Von besonders ausführlichen und intensiven Untersuchungen an Alpengletschern zeugen die Arbeiten von B. MESSERLI et al. (1975) und H.J.ZUMBÜHL (1975 und 1980) am Unteren und Oberen Grindelwaldgletscher. In den Gebirgen zwischen Mittelmeer und Äquator werden Verbreitung und Höhenstufung der Glazial- und Periglazialformen und -prozesse von der letzten Kaltzeit bis zur Gegenwart klimageschichtlich untersucht (B. MESSERLI 1980, B. MESSERLI, M. WINIGER U. P. ROGNON 1980).

H. LESER und Mitarbeiter vom Geographischen Institut der Universität Basel bemühen sich u.a. mit der geomorphologischen Kartierung der Schweiz im Maßstab 1: 25000 (H. LESER U. J. P. PORTMANN, O. J.).

Vom Geographischen Institut der ETH hat sich F. MACHATSCHEK (1928) mit Fragen der Talbildung in der Innerschweiz und in Graubünden beschäftigt. Am Geologischen Institut der ETH hat R. ST AUB (1934) die sich aus der Tektonik ergebenden Fakten zu seinem Werk "Grundzüge und Probleme alpiner Morphologie» zusammengefaßt.

Derzeit werden Lignit-Vorkommen am Genfersee von der Universität Lausanne aus neu bearbeitet.

Geographen der Universität Zürich arbeiten an einer Geschichte der Gletscherschwankungen im Alpenraum und dessen Vorland. Davon zeugen die Dissertationen von W.SCHNEEBELI (1976) und F.RÖTHLISBERGER (1976). F. RÖTHLISBERGER untersuchte erstmals fossile Bäume radio-dendroklimatisch. Während die beiden Erstgenannten im Wallis arbeiteten, befaßte sich F. BEELER (1977) mit geomorphologischen Untersuchungen am Spät- und Postglazial im Schweizerischen Nationalpark und im Berninapaß-Gebiet.

Seit über dreißig Jahren werden am selben Geographischen Institut - zuerst unter H. BOESCH (1911-1978), dann unter G.FURRER - geomorphologische Untersuchungen vorwiegend im Raume des nördlichen und nordöstlichen Mittellandes durchgeführt. Arbeiten über ganze Flußtäler, so z.B. das Glattal (M.STEIN, 1949) oder Teile desselben (E. SOMMERHALDER, 1968), sowie über das Zürichseetal (H.RINGGER, 1964) oder das Dättnau (N.KAISER, 1979) bilden Schwerpunkte unserer Forschung wie Studien über spezielle thematische Gebiete, so z.B. Löß-Stratigraphie (H.GoudA, 1962) und periglaziale Formen (F.BACHMANN, 1966) sowie Verwitterungsuntersuchungen auf Schotterterrassenflächen (T. STRÜBY, 1969). Einen weiteren Themenkreis bildet die Geschichte des ehemaligen Rhein-Gletschers. Hier sind es vor allem die Arbeiten zur Erhellung der Litho- und Chronostratigraphie, die zur Vertiefung des Sachverhaltes wesentlich beigetragen haben (A.LEEMANN, 1957; w. OERTLE, 1954; L. ELLENBERG, 1972; W.A.KELLER, 1977; U. KAESER, 1980). Auch außerhalb des eigentlichen Rheingletschersystems gelangte man zu neuen Erkenntnissen, so z. B. im nordöstlichen Aargau (E. BUGmanN, 1956) oder im zentralen westlichen Mittelland (H. ZIMMERMANN, 1961), aber auch - aufgrund einer mehr technischen Fragestellung - über den Einfluß der Limnologie auf das Gerinnebett der Thur wurde gearbeitet (B.MÖRGELI, 1974). Im Voralpengebiet konnten einige Beiträge zur Morphogenese der entsprechenden Räume geliefert werden (H.ANDRESEN, 1962; G.JUNG, 1969; O. KELLER, 1973， 1981; O. KELLER U. E. KRAYSS, 1980).

1979 und 1981 erschienen Arbeiten von C. A.BURGA zum Quartär des Schams und des San BernardinoPaßgebietes; 1981 jene von J. SUTER zur Gletschergeschichte des Oberengadins und von M. MAISCH, der besonders Beiträge zum Spätglazial zwischen Landwasser- und Albulatal lieferte.

Laufende Arbeiten sind der jüngsten Vergangenheit des Aletschgletschers (H.P. HOLZHAuSER, der historische Schrift- und Bildquellen sowie fossile Bäume und ehemalige Wasserleitungen auswertet), dem Postund Spätglazial im Saastal (w. BIRCHER) und besonders dem Postglazial im Gotthardgebiet mit radio-dendroklimatischer Auswertung fossiler Bäume gewidmet (F. RENNER).

Im Dättnauer Trockental bei Winterthur gelang N. KAISER (1979) die Freilegung eines späteiszeitlichen Birken-/Föhrenwaldes.

Die radio-dendrochronologische Auswertung der rezenten und fossilen Holzproben des Zürcher Geographischen Instituts erfolgt durch Studenten und 
Doktoranden desselben an der Eidg. Anstalt für das forstliche Versuchswesen in Birmensdorf (F.H.SCHWEINGRUBER). Routinemäßige RadiokarbonDatierungen werden einerseits am Geographischen Institut der Universität Zürich (w. A. KeLLER) und andrerseits am Physikalischen Institut der Universität Bern (Prof. H. OESCHGER) durchgeführt, wo außerdem zum Problem dieser absoluten Altersbestimmung Forschung betrieben wird. H.OESCHGER, U.EICHER, U. SIEgenthaleR und weitere Mitarbeiter des Berner Physikalischen Instituts sind mit intensiven Untersuchungen des klimaempfindlichen ${ }^{18} O-\lambda^{16} O$-Verhältnisses in Seekreide beschäftigt. An den Übergängen Älteste Dryas/Bølling, Allerød/Jüngere Dryas sowie Jüngere Dryas/Präboreal wurden in zahlreichen Seekreideprofilen signifikante Veränderungen dieses Isotopenverhältnisses festgestellt (U.EICHER U. U. SIEGENTHALER 1976; H.OESCHGER 1980; H.OESCHGER, M. WELTEN, U.EICHER, M.MÖLL, T.RIESEN, U. SIEGENTHALER U. S. WEGMÜLLER 1980; U. EICHER, U. SIEGENTHALER U. S. WEGMÜLLER 1981).

Zur paläoklimatologischen Auswertung quartärer Mollusken aus dem Genfer Raum und den Alpen sind beispielsweise die Arbeiten von L. CHAIX u. PH. OLIVE (1980) und L. ChaIX, B. CHASSAING U. PH. OLIVE (1982) zu erwähnen.

M.GAMPER (1981) nahm vor einigen Jahren die Arbeiten von G. FURRER und seinen Schülern wieder auf (vgl. z. B.: H. ELSASSER 1968, K. GRAF 1971). Dabei wird versucht, Wechsellagerungen von fossilen Böden mit Solifluktionsschutt klimamorphologisch zu deuten. Die aus paläoklimatischen Gründen wenig verbreiteten Lößvorkommen der Schweiz machen es verständlich, daß die Lößforschung ein eher kümmerliches Dasein fristet; P. FITZE bearbeitet Lösse in der Nordostschweiz.

Aus Messungen der Klimaelemente an zahlreichen Beobachtungsorten sollen Rekonstruktionen des Klimas möglich werden. Vertreter des Geographischen Instituts der Universität Zürich bearbeiten an der Schweizerischen Meteorologischen Anstalt (SMA) alte Klimadaten (M. SCHÜEPP und O.GISLER). Die Klimareihen reichen heute bis zur Mitte des 18. Jahrhunderts zurück. Mit aufgearbeiteten und miteinander vergleichbaren Zahlenwerten wird eine Interpretation der atmosphärischen Verhältnisse derart möglich sein, daß vergleichbare klimageschichtliche Untersuchungen angeknüpft werden können.

Eine aus je drei Mitgliedern bestehende gemeinsame Kommission des $\mathrm{SAC}^{3}$ und der SNG nahm 1874 die Vermessung des Rhonegletschers auf. Seit 1880 liegen jährlich Daten über Zungen-Längenänderungen von verschiedenen Gletschern vor. Heute werden von der 1892 gegründeten Gletscherkommission der SNG 120 Alpengletscher beobachtet.

\footnotetext{
${ }^{3}$ Schweizer Alpen-Club
}

\subsection{Paläobotanik (Übersicht zur pollenanalytischen Forschung)}

Während die Vegetationsgeschichte sich zunächst nur auf Großreste - Blätter, Stengel, Früchte, Samen, Rinden und Wurzeln - abstützen konnte, erlaubte erst die Pollenanalyse in Verbindung mit Großresten, eine Entwicklungsgeschichte zu schreiben (vgl. w. RYTZ 1930, 1949). Immerhin konnten O. HEER, w. RYTZ und A. JaCCARD (in E. BaUmberger et al., 1923) die Floren der Schieferkohlen, der Glazialfloren (A. G. NATHORST, 1874, 1919; C. SCHRÖTER, 1883), E. NEUWEILER (1905, $1935,1946)$ jene der Pfahlbau-Siedlungen und J. FRÜH u. C. SCHRÖTER (1904) jene der schweizerischen Moore bereits genau erfassen.

Die ersten pollenanalytischen Untersuchungen in der Schweiz wurden an einigen Mooren des Juras von H.SPINNER (1925) und am Hallwilersee von C. TROLL durchgeführt (publiziert von H. HÄRRY 1925). 1926 und 1928 veröffentlichte P. KELLER erste umfassende pollenanalytische Untersuchungen zahlreicher Moore der Schweiz. Insgesamt 26 Moore bzw. Pfahlbauplätze aus dem Mittelland, den Voralpen und dem Jura bearbeitete er in seiner 1928 veröffentlichten Dissertation. Er entwarf eine auf pollenanalytischen Ergebnissen beruhende postglaziale Vegetationsgeschichte der Schweiz. Es folgten kurz nacheinander mehrere pollenanalytische Arbeiten von E. FURRER (1927), W. LÜDI (1929), M. WELTEN (1929), w. RYTZ (1931).

Die pollenanalytische Forschung konzentrierte sich zunächst praktisch ausschließlich auf vegetationsgeschichtliche Fragen. Anfangs der fünfziger Jahre gelangte der Gesichtspunkt des Vegetationswandels als Ergebnis von klimatischen Schwankungen ins Diskussionsfeld der Paläobotaniker, so z. B. in Arbeiten von M. WELTEN (1944, 1952, 1958), w.LÜDI (1954) und H. ZOLLER $(1958,1960)$. H. ZOLLER publizierte 1960 und 1966 erstmals die von ihm auf pollenanalytischem Wege erkannten postglazialen Klimaschwankungen der Alpen. M. WelteN und seine Schüler beschäftigten sich U. a. mit geochronologischen Problemen (M. WELTEN 1944, 1947, 1972, S. WEgMÜLLER U. M. WELTEN 1973, M. KÜTTEL 1977, U. EICHER 1979). Die pollenanalytische Forschungstätigkeit von H. zOLLER (Basel) und seiner Schüler hat als Schwerpunkt das Spät- und Postglazial der Zentral- und Südalpen, insbesondere Graubündens (z.B. C. BURGA 1980, C. HEITZ 1975, L.KING 1974, H.KLEIBER 1974, H.-J.MÜLLER 1972). Ferner besteht eine Zusammenarbeit mit archäologischen Projekten (A. K. HEITZ 1976, 1978; S. JACOMET 1980). Die Forschergruppe von M. WELTEN und neuerdings G.LANG (Bern) weist eine vielfältige Betätigung in den Vor-, Zentral- und Südalpen sowie im Jura und Mittelland auf (z. B. B. AMMANN 1975, K. AMMANN 1976, R. HäNI 1964, K. HEEB U. M. WELTEN 1972, M. KÜTTEL 1979, V. MARKGRAF 1969, R. SCHNEIDER 1978, H.P. WEGMÜLLER 1976, s. WEGMÜLLER 1966). Der Erforschung 
des Eem-Interglazials und der letzten Eiszeit im schweizerischen Mittelland wurde durch die Untersuchungen von M. WELTEN $(1979,1981,1982)$ besondere Beachtung geschenkt.

Ferner werden pollenanalytische Untersuchungen an Hochschulinstituten in Lausanne (P. U. M. viLLARET, M.J.GaIllaRD), Neuchâtel (F.MATTHEY) und Zürich betrieben.

Im Genfer Becken versucht c. REYNAUD (1979, 1981, 1982, 1983) die Abfolgen vor der Alluvion ancienne pollenanalytisch und damit paläoklimatisch und chronostratigraphisch zu gliedern.

\subsection{Paläozoologie}

Im ersten Band der «Urgeschichte der Schweiz» (von O. TSCHUMI 1949 herausgegeben) gaben K. HESCHELER und E.KUHN einen ausführlichen und mit einem umfassenden Literaturverzeichnis versehenen Überblick über den Wissensstand der Quartärpaläontologie in der Schweiz vor 1950. Später erfolgten in der von W. DRACK redigierten Reihe "Ur- und frühgeschichtliche Archäologie der Schweiz" knappe Übersichten unter Berücksichtigung neuerer Arbeiten, so z.B. von E. KUHN-SCHNYDER (1968) und von H.HARTMANNFRICK (1969). Seitdem wurden zahlreiche bemerkenswerte Einzelfunde publiziert, wie beispielsweise von E. SCHмID (1969).

H. R. STAMPFLI lieferte zahlreiche Arbeiten über steinzeitliche Osteologica von überregionaler Bedeutung.

Die meisten neueren Veröffentlichungen betreffen postglaziale Faunen bzw. Faunenelemente (F. u. M. BURRI 1977, v. AELLEN 1978). R. HANTKE faßte 1978 die neuesten Ergebnisse zur eiszeitlichen Fauna der Schweiz zusammen.

\subsection{Ur- und frühgeschichtliche Archäologie}

Die Forschung der ur- und frühgeschichtlichen Archäologie in der Schweiz weist im wesentlichen zwei Schwerpunkte auf: 1. Seeufer-Siedlungen und Seespiegelschwankungen sowie 2. Untersuchungen im alpinen Raum (besonders im Wallis und in Graubünden). Das am umfangreichsten publizierte Untersuchungsmaterial einer Ufersiedlung stammt von Twann am Bielersee ("Die neolithischen Ufersiedlungen von Twann"; Schriftenreihe der Erziehungsdirektion des Kantons Bern, herausgegeben vom Archäologischen Dienst des Kantons Bern, 19 Bände). Den zahlreichen ur- und frühgeschichtlichen
Siedlungsplätzen am Neuenburgersee widmet sich u. a. die luftbildarchäologische Untersuchung von M. EGLOFF (1981). Aufgrund der durch Erosion freigelegten Pfähle wird versucht, ehemalige Siedlungsstrukturen zu rekonstruieren.

Im Bereich des unteren Zürichsees wurden in letzter Zeit bedeutende jungneolithische und bronzezeitliche Funde getätigt, die auf ihre ökonomischen und chronologischen Aspekte hin ausgewertet wurden (U. RUOFF U. A.-C. KUSTERMANN 1982, U.RUOFF 1981, S. JACOMET 1980, M. PRimas 1981, M. Joos 1976). Pollenanalytische Untersuchungen in den Sedimenten der Seerandsiedlungen führten u.a. zum Schluß, daß in der Umgebung des unteren Zürichsees im Neolithikum nur kleine Waldflächen gerodet wurden (A. K. HEITZ 1978).

In jüngster Zeit konnten frühbronzezeitliche Siedlungsstrukturen (Reihenhäuser) unter der Mozartstraße in Zürich aufgedeckt werden. Unter der Leitung des Stadtarchäologen, U.RUOFF, der ein eigenes Labor für Dendrochronologie im Büro für A rchäologie der Stadt Zürich unterhält, sind vielfältige Untersuchungen der Kulturschichten aus sechs Hauptsiedlungsperioden im Gange.

$\mathrm{Zu}$ den Ufersiedlungen des Bodensees haben erst seit kurzem die Bestandesaufnahmen begonnen.

Die Untersuchungen von Seeufersiedlungen ergeben vielfältige, zeitlich gestaffelte Hinweise siedlungsgeographischer und kulturgeschichtlicher Art zum jüngsten Abschnitt des Quartärs. Bemerkenswert sind die deutlich sich abzeichnenden Siedlungskonzentrationen rund um den Neuenburgersee und am Unterende des Zürichsees in der Zeit von 1100 bis 800 v. Chr. Einen echten quartärgeologischen Beitrag liefern auch die Untersuchungen zu den holozänen Seespiegelschwankungen (w.LÜDI 1935, B.AMMANN 1975, C.SCHINDLER 1981, M. Joos 1982).

Ur- und frühgeschichtliche Forschungen im alpinen Raum befassen sich u.a. mit der Frage der Landnahme durch den Menschen.

Noch liegen wenige Ergebnisse zur mesolithischen Besiedlung der Alpen vor (J. F. BERGIER 1979). Von der Universität Genf aus erfolgen archäologische Untersuchungen im unteren Wallis (M. R. SAUTER, A. GALLAY U. L. CHAIX 1971; zu der Steinkisten-Nekropole von Sion vgl. O.-J. BOCKSBERGER, herausgegeben von A. GALLAY, Genf, 4 Bände, 1976 und 1978).

Aus dem Gebiet Graubündens sind zu erwähnen die sehr aufschlußreichen Grabungsberichte von J.RAGETH (1981) über die bronzezeitliche Siedlung auf dem Padnal bei Savognin (aufgrund von Plattenschlacken-Funden laufen Untersuchungen zur lokalen Erzverhüttung) und die noch nicht abgeschlossenen Grabungen auf dem Petrushügel bei Cazis (neolithischer Siedlungsplatz) durch M.PRIMAS in Zürich. 
Für die vorliegende Publikation wurden in verdankenswerter Weise wesentliche Beiträge beigesteuert von:

Kap. 3.1 Geologie:

Ch.Schlüchter, Zürich/C.Schindler, Zürich/W.K. Nabholz, Bern

Kap. 3.2 Geomorphologie, Dendrochronologie und Klimatologie:

B. Messerli, Bern/H. Oeschger, Bern/W. A. Keller, Zürich/O. Gisler, Zürich/L. Chaix, Genève

\section{Ausgewählte Literatur}

AMMANN B. (1975): Vegetationskundliche und pollenanalytische Untersuchungen auf dem Heidenweg im Bielersee. In: Beitr. Geobot. Landesaufn. d. Schweiz, 56: 1-76.

AMMANN K. (1976): Der Oberaargletscher im 18., 19. und 20. Jahrhundert. In: Z. f. Gl.kde. u. Glaz.geol., 12, 2: 253-291.

BACHMANN F. (1966): Fossile Strukturböden und Eiskeile auf jungpleistozänen Schotterflächen im nordostschweizerischen Mittelland. Diss. Univ. Zürich. Kunz, Pfäffikon/ZH, 176 Seiten.

BEELER F. (1977): Geomorphologische Untersuchungen am Spät- und Postglazial im Schweizerischen Nationalpark und im Berninapaßgebiet (Südrätische Alpen). Diss. Univ. Zürich. Erg. wiss. Unters. Schweiz. Nat.park, 15, 77: 131-276.

BERGIER J.-F. (Hrsg.) (1979): Geschichte der Alpen in neuer Sicht. In: Schweiz. Ztschr. f. Gesch., 29, 1; Schwabe, Basel, 300 Seiten.

BURGA C.A. (1981): Glazialmorphologische Untersuchungen im Hinterrhein-Tal und am Bernhardin-PaB. In: Vjschr. Natf. Ges. Zürich, 126, 4: 237-267.

CHAIX L. U. OLIVE PH. (1980): Paléoclimatologie continentale et mollusques dulcicoles. $8^{\mathrm{e}}$ réunion annuelle des sciences de la terre, Marseille 1980, Paris, S. 90.

EGLOFF M. (1979): La transition du tardiglaciaire au postglaciaire en Suisse. Colloques intern. C.N.R.S., La fin des temps glaciaires en Europe, Nr. 271: 231-237.

EGLOFF M. (1981): Versunkene Dörfer der Urnenfelderzeit im Neuenburger See. (Forschungen der Luftbildarchäologie). In: Archäolog. Korr.blatt: 55-63.

EICHER U. (1979): Die ${ }^{18} \mathrm{O} /{ }^{16} \mathrm{O}$ - und ${ }^{13} \mathrm{C} /{ }^{12} \mathrm{C}$-Isotopenverhältnisse in spätglazialen Süßwasserkarbonaten und inr Zusammenhang mit den Ergebnissen der Pollenanalyse. Diss. Univ. Bern. Universitätsdruckerei Bern, 205 Seiten.

EICHER U. U. SIEGENTHALER U. (1976): Palynological and oxygen isotope investigations on Late-Glacial sediment cores from Swiss lakes. Boreas, 5: 109-117.

FURRER G. (1965): Die Höhenlage von subnivalen Bodenformen, untersucht in den Bündner und Walliser Alpen und verglichen mit den Verhältnissen im oberen Braldo- und Biafotal (Karakorum). Habilitationsschrift Univ. Zürich, Kunz, Pfäffikon/ZH, 89 Seiten.

GAMPER M. (1981): Heutige Solifluktionsbeiträge von Erdströmen und klimamorphologische Interpretation fossiler Böden. Diss. Univ. Zürich. Erg. wiss. Unters. Schweiz. Nat.park, 15, 79: 355-443.
Kap. 3.4 Paläozoologie:

K. A. Hünermann, Zürich

Kap. 3.5 Ur- und frühgeschichtliche Archäologie: M. Primas, Zürich/M. Joos, Basel/M. Egloff, Neuchâtel

Spezieller Dank gilt R. Hantke, Zürich, für die kritische Durchsicht des Manuskripts und für seine Beiträge zu den Kapiteln 1, 2, 3.1-3.4.

HÄBERLI W. (1975): Untersuchungen zur Verbreitung von Permafrost zwischen Flüelapaß und Piz Grialetsch (Graubünden). In: Mitt. Vers.anst. f. Wasserbau, Hydrolog. u. Glaziol., Nr. 17, 221 Seiten.

HANTKE R. (1978), (1980), (1983): Eiszeitalter. Ott, Thun, Bde. 1,2 u. 3.

HEIM ALB. (1885): Handbuch der Gletscherkunde. Stuttgart, 560 Seiten.

HEITZ A.K. (1978): Pollenanalytische Untersuchungen an den neolithischen und spätbronzezeitlichen Seerandsiedlungen "Kleiner Hafner", "Großer Hafner" und "Alpenquai" im untersten Zürichsee (Schweiz). In: Bot. Jahrb. Syst. 99, 1: 48-107.

HESCHELER K. U. KUHN E. (1949): Kap. “Die Tierwelt". In: Tschumi O. 1949: Urgeschichte der Schweiz, Bd.1.

JACOMET S. (1980): Botanische Makroreste aus den neolithischien Seeufersiedlungen des Areals "Pressehaus Ringier" in Zürich (Schweiz). Stratigraphische und vegetationskundliche Auswertung. In: Vjschr. Natf. Ges. Zürich. 125, 2: 73-163.

JÄCKLI H. (1962): Die Vergletscherung der Schweiz im Würmmaximum. In: Eclogae Geol. Helvet. 55, 2: 285-294.

JOOS M. (1976): Die Sedimente der neolithischen Station Feldmeilen-Vorderfeld. In: Feldmeilen-Vorderfeld. Die Ausgrabungen 1970/1971. Antiqua 5: 103-132, Basel.

JOOS M. 1982: Swiss Midland-lakes and climatic changes. In: A. F. Harding (Edit.), (1982): Climatic change in Later Prehistory. University press, Edinburgh, S. 44-51.

KÄSER U.J. (1980): Glazialmorphologische Untersuchungen zwischen Töss und Thur. Diss. Univ. Zürich, Juris, Zürich, 135 Seiten.

KAISER K. (1975): Die Inlandeis-Theorie, seit 100 Jahren fester Bestand der Deutschen Quartärforschung. Eiszeitalter u. Gegenwart, 26, 1-30.

KAISER N. (1979): Ein späteiszeitlicher Wald im Dättnau bei Winterthur/Schweiz. Diss. Univ. Zürich. Ziegler, Winterthur, 90 Seiten.

KELLER P. (1928): Pollenanalytische Untersuchungen an Schweizer Mooren und inre florengeschichtliche Deutung. Diss. ETH, Veröff. Geobot. Inst. Rübel in Zürich, 5: 1-163.

KELLER W.A. (1977): Die Rafzerfeldschotter und ihre Bedeutung für die Morphogenese des zürcherischen Hochrheingebietes. Diss. Univ. Zürich. Vjschr. Natf. Ges. Zürich, 122, 3 : 357-412. 
KING L. (1974): Studien zur postglazialen Gletscher- und Vegetationsgeschichte des Sustenpasses. Diss. Univ. Basel. Basler Beitr. z. Geographie, 18: 1-123.

KLEBELSBERG VON R. (1949): Handbuch der Gletscherkunde und Glazialgeologie. Springer, Wien; 2 Bde., 404 u. 623 Seiten.

KUHN-SCHNYDER E. (1968): Die Tierwelt des Pleistozäns und Alt-Holozäns. In: Ur- und frühgeschichtliche Archäologie der Schweiz. Bd. 1.

LESER H. (1981/82): Die Geomorphologie und die Schweizerische Geomorphologische Gesellschaft. In: Geograph. Taschenb. 1981/82, Wiesbaden, 45-58.

LESER H. U. PORTMANN J.P. O.J.: Die geomorphologische Kartierung in der Schweiz. Memorandum der Schweiz. Geom. Gesellschaft, “Arbeitskreis GMK $25 \mathrm{CH}$ ”, 25 Seiten.

MAISCH M. (1981): Glazialmorphologische und gletschergeschichtliche Untersuchungen im Gebiet zwischen Landwasserund Albulatal (Kt. Graubünden, Schweiz). Diss. Univ. Zürich. Physische Geographie, 3: 1-215.

MESSERLI B. (1980): Die afrikanischen Hochgebirge und die Klimageschichte Afrikas in den letzten 20000 Jahren. In: Oeschger H., Messerli B. U. Svilar M. (Hrsg.), Das Klima. Springer, Berlin, S. 64-90.

MESSERLI B. et al. (1975): Die Schwankungen des Unteren Grindelwaldgletschers seit dem Mittelalter. Z. f. Gl.kde u. Glaz.geol., 11, 1: 3-110.

MESSERLI B., WINIGER M. u. ROGNON P. (1980): The Saharan and East African uplands during the Quaternary. In: M.A.J.Williams U. H. Faure (Ed.) 1980: The Sahara and the Nile. Quaternary environments and prehistoric occupation in northern Africa. A. A. Balkema, Rotterdam, S. 87-132.

OESCHGER H. (1980): In der Natur gespeicherte Geschichte von Umweltsvorgängen. In: H. Oeschger, Messerli B. u. Svilar M. (Edit.) 1980: Das Klima, S. 209-236.

OESCHGER $\mathrm{H}$. et al. (1980): ${ }^{14} \mathrm{C}$ and other parameters during the Younger Dryas cold phase. Radiocarbon, 22, 2: 299-310.

PENCK A. u. BRÜCKNER E. (1909): Die Alpen im Eiszeitalter. Tauchnitz, Leipzig; 3 Bde.

PFISTER C. (1981): Die Fluktuationen der Weinmosterträge im schweizerischen Weinland vom 16. bis ins frühe 19. Jahrhundert. Klimatische Ursachen und sozioökonomische Bedeutung. Schweiz. Ztschr. f. Geschichte, 31: 445-491.

PFISTER C. (im Druck): Das Klima der Schweiz von 1525 bis 1860 und seine Bedeutung in der Geschichte von Bevölkerung und Landwirtschaft.

PRIMAS M. (1981): Urgeschichte des Zürichseegebietes im Überblick: Von der Steinzeit bis zur Früheisenzeit. In: Zürcher Seeufersiedlungen - Von der Pfahlbauromantik zur modernen archäologischen Forschung. Helvetia archaeologica, Heft 45/48: 5-18

RAGETH J. (1981): Die bronzezeitliche Siedlung auf dem Padnal bei Savognin (Oberhalbstein GR). Grabung 1977. In: Jb. d. Schweiz. Gesellsch. f. Ur- und Frühgesch., 64: 27-71.

RÖTHLISBERGER F. (1976): Gletscher- und Klimaschwankungen im Raum Zermatt, Ferpècle und Arolla. Die Alpen, 52, 3/4: 59-152.

RUOFF U. (1981): Die Entwicklung der Unterwasserarchäologie im Kanton Zürich. In: Zürcher Seeufersiedlungen - Von der Pfahlbauromantik zur modernen archäologischen Forschung. Helvetica archaeologica, Heft 45/48: 62-70.

RUOFF U. u. KUSTERMANN A.-C. (1982): Die jungneolithische Pfyner-Gruppe im unteren Zürichseebecken. Moreland, Bad Bramstedt.
RYTZ W. (1930): ;Neue Wege in der prähistorischen Forschung mit besonderer Berücksichtigung der Pollenanalyse. Mitt. Antiquar. Ges. in Zürich, 30, 7: 58-77.

SAUTER M. R., GALLAY A. u. CHAIX L. (1971): Le néolithique du niveau inférieur du Petit-Chasseur à Sion, Valais. In: Jb. d. Schweiz. Gesellsch. f. Ur- u. Frühgesch., 56: 17-76.

SCHINDLER C. (1976): Eine geologische Karte des Zürichsees und ihre Deutung. Eclogae geol. Helv., 69, 1: 125-138.

SCHINDLER C. (1981): Geologische Unterlagen zur Beurteilung archäologischer Probleme in den Seeufergebieten. Helvetia archaeologica, Heft 45/48: 71-88.

SCHLÜCHTER C. (1976): Geologische Untersuchungen im Quartär des Aaretals südlich von Bern (Stratigraphie, Sedimentologie, Paläontologie). Diss. Univ. Bern. Beiträge z. Geol. Karte d. Schweiz. N. F., 148: 1-118.

SCHLÜCHTER C. (1980): Remarques sur les subdivisions lithostratigraphiques du Quaternaire au nord des Alpes Centrales (Suisse). In: Problèmes de stratigraphie quaternaire en France et dans les pays limitrophes. J. Chaline (Edit.), Suppl. au Bull. de l'Afeq, N. S., 1: 22-30.

SCHLÜCHTER C. (1981): Remarks on the Pleistocene morphogenetic evolution of the Swiss Plain. Z. Geomorph. N.F., 40 (Suppl.-Bd.): 61-66.

SCHMID E. (1958): Höhlenforschung und Sedimentanalyse. Ein Beitrag zur Datierung des Alpinen Paläolithikums. Inst. f. Ur- u. Frühgesch. der Schweiz, Basel.

SCHNEEBELI W. (1976): Untersuchungen von Gletscherschwankungen Val de Bagnes. Die Alpen, 52, 3/4: 5-57.

SUTER J. (1981): Gletschergeschichte des Oberengadins: Untersuchung von Gletscherschwankungen in der Err-JulierGruppe. Diss. Univ. Zürich, Physische Geographie, 2: 1-147.

WEGMÜLLER S. u. WELTEN M. (1973): Spätglaziale Bimstufflagen des Laacher Vulkanismus im Gebiet der westlichen Schweiz und der Dauphiné (F). Eclogae geol. Helvet. 66, 3: 533-541.

WELTEN M. (1952): Über die spät- und postglaziale Vegetationsgeschichte des Simmentals. Veröff. Geobot. Inst. Rübel in Zürich, 26: 1-135.

WELTEN M. (1979): Eis, Wasser und Mensch haben das Aaretal verändert. Ergebnisse von 50 Jahren Pollenanalyse in Bern. Mitt. Natf. Ges. Bern, N. F. 36: 17-40.

WELTEN M. (1981): Gletscher und Vegetation im Lauf der letzten hunderttausend Jahre. Vorläufige Mitteilung. Jb. Schweiz. Natf. Ges., wiss. Teil, 5-18.

WELTEN M. (1981): Verdrängung und Vernichtung der anspruchsvollen Gehölze am Beginn der letzten Eiszeit und die Korrelation der Frühwürm-Interstadiale in Mittel- und Nordeuropa. Eiszeitalter u. Gegenwart, 31, 187-202.

ZOLLER H. (1960): Pollenanalytische Untersuchungen zur Vegetationsgeschichte der insubrischen Schweiz. Denkschr. Schweiz. Natf. Ges., 83, 2: 45-156.

ZOLLER H., SCHINDLER C. U. RÖTHLISBERGER H. (1966): Postglaziale Gletscherstände und Klimaschwankungen im Gotthardmassiv und Vorderrheingebiet. Verh. Natf. Ges. Basel, 77, 2: 97-164.

ZUMBÜHL H.J. (1975): Die Schwankungen des Unteren Grindelwaldgletschers in den historischen Bild- und Schriftquellen des 12. bis 19. Jahrhunderts. Z. f. Gl.kde u. Glaz.geol., $11,1: 12-50$.

ZUMBÜHL H.J. (1980): Die Schwankungen der GrindelwaldGletscher. Denkschr. Schweiz. Natf. Ges., 92, 279 Seiten. 